\title{
Focus on neurotechnologies
}

$\mathrm{N}$ eurotechnologies have come far in just a few years, and we can expect them to provide new approaches to shed light on brain function and contribute novel therapeutic interventions. In basic neuroscience, a diversity of molecular approaches are enabling researchers to track and manipulate the activity of specific neuronal populations in the brain and peripheral nervous systems of freely moving mice, and single-cell 'omics' is allowing profound characterization of brain cells, which will provide a finer understanding of neuroanatomy and function in health and disease. In animal models, a range of exciting electrical, chemical and optical tools continue to emerge, serving as fundamental tools for neuroscientists and informing study design in humans (see Review by Frank et al.).

In the clinic, although surgical and small-molecule approaches traditionally have provided treatments for neurological and psychiatric disorders, these remain blunt therapeutic instruments, often accompanied by considerable side effects in return for limited efficacy. Today, electrode arrays allowing the recording and stimulation of neurons with a high degree of precision are being engineered to be flexible and soft, injected into deep-brain structures with a syringe, and connected to decoders and actuators. This is opening new opportunities for restoring lost function by allowing control of robotic arms in quadriplegia patients. Deep brain stimulation, meanwhile, is an effective treatment for common movement disorders, including Parkinson's disease, but is not without side effects. Although

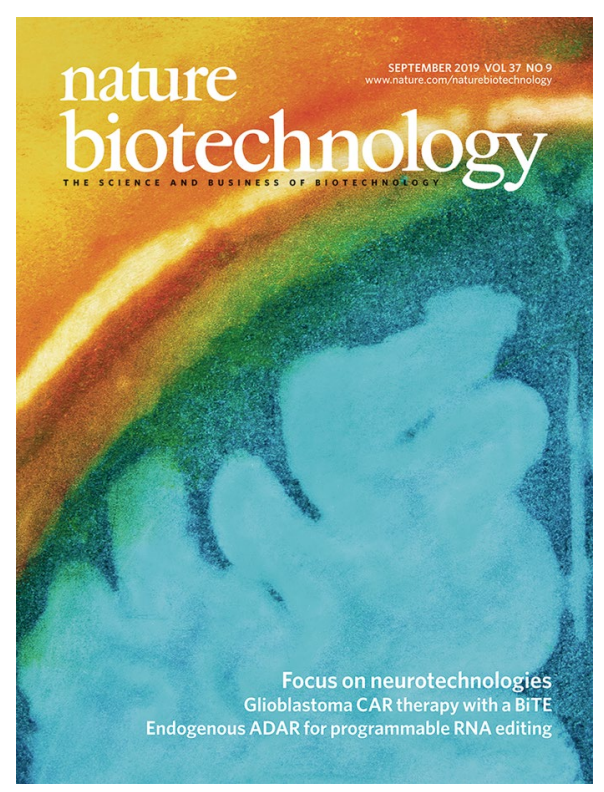

A vast number of patients could benefit from precision therapies for psychiatric and neurological diseases, and an improved mechanistic understanding of brain connectivity and function is key to support their development and application (see Editorial). Brain-computer interfaces best exemplify the type of strategies that are likely to become more broadly used in the coming years-implanted devices record brain signals, which are decoded using complex algorithms and translated into actions using prosthetics (see News Feature by Smalley). Brain-machine interfaces have been successfully piloted in a couple of dozen individuals, and the field is pushing on towards safer and better systems (see Patent Table).

Non-invasive approaches to measure and modulate brain function, such as transcranial magnetic stimulation, are effective in treating disease and are also gaining traction in the direct-toconsumer world (see News Feature by Waltz).

As neurotechnology development moves full steam ahead, it will be important to continue discussions and take appropriate actions with regards to the ethical issues surrounding the use of neurotechnologies for the treatment of disease (see Q\&A) as well as in the direct-to-consumer space (see Correspondences by Wexler and by Ienca et al.).

Irene Jarchum

Published online: 4 September 2019 https://doi.org/10.1038/s41587-019-0261-5 